\title{
Fidelity for multimode thermal squeezed states
}

\author{
Gh.-S. Paraoanu* \\ Department of Physics, University of Illinois at Urbana-Champaign, 1110 W. Green St., Urbana, IL 61801, USA; e-mail: \\ paraoanu@physics.uiuc.edu \\ Horia Scutaru \\ Department of Theoretical Physics, Institute of Atomic Physics, PO BOX MG-6, R-76900 Bucharest-Magurele, Romania; \\ e-mail: scutaru@theor1.theory.nipne.ro
}

In the theory of quantum transmission of information the concept of fidelity plays a fundamental role. An important class of channels, which can be experimentally realized in quantum optics, is that of Gaussian quantum channels. In this work we present a general formula for fidelity in the case of two arbitrary Gaussian states. From this formula one can get a previous result [迎, for the case of a single mode; or, one can apply it to obtain a closed compact expression for multimode thermal states. The concept of fidelity used in this paper is the standard one 24. It can be defined by

$$
F\left(\rho_{1}, \rho_{2}\right) \stackrel{\text { def }}{=} \max _{\left.\left|\psi_{1}>,\right| \psi_{2}\right\rangle}\left|\left\langle\psi_{1} \mid \psi_{2}\right\rangle\right|^{2}
$$

where $\left|\psi_{i}\right\rangle, i=1,2$ are purifications of the density matrices $\rho_{i}$.

03.65.Bz, 42.50.Dv, 89.70.+c

\section{INTRODUCTION}

Within recent years, the quantum theory of information, an extension of the classical theory of information to the quantum realm, has emerged as a fascinating research field. A great deal of effort has been devoted to the issue of transmitting a state through noisy quantum channels despite the quantum-mechanical uncertainties in our knowledge about that state. This is a different problem from the classical situation, where the states are mutually exclusive and the input system may remain in its initial state; in the quantum case the states are density operators on a Hilbert space and the non-cloning theorem [5] precludes the input system, in general, to retain its original state. Moreover, for a noisy quantum channel the state is subject to a decoherence process, due to the interaction with an external environment, which further decreases the reliability of information processing. Thus, a fundamental problem is to extend the classical encoding and decoding procedures to quantum channels

\footnotetext{
* On leave from the Department of Theoretical Physics, Institute of Atomic Physics, PO Box MG-6, R-76900, BucharestMagurele, Romania.
}

and to define an upper limit (the channel capacity) to the amount of quantum information that can be transmitted with an arbitrary high fidelity. Recently, work [6] has been focused on quantum channels which use Gaussian (or quasi-free, [7, 8]) states for the transmission of information. In this case, a first issue to be raised is how to calculate the fidelity given two mixed Gaussian states; partial answers, depending on the particular type of mixtures under consideration have already been given in the literature [1,9, 10]. In this paper, we will give a general formula for the fidelity of two quasi-free states, and show that the previous results can be obtained as particular cases. The formula can also be applied to some interesting cases, such as two-mode systems [11].

Let $\rho_{1}$ and $\rho_{2}$ two density operators which describe two mixed states. The transition probability $P\left(\rho_{1}, \rho_{2}\right)$ has to satisfy the following natural axioms:

1. $P\left(\rho_{1}, \rho_{2}\right) \leq 1$ and $P\left(\rho_{1}, \rho_{2}\right)=1$ if and only if $\rho_{1}=$ $\rho_{2} ;$

2. $P\left(\rho_{1}, \rho_{2}\right)=P\left(\rho_{2}, \rho_{1}\right)$;

3. If $\rho_{1}$ is a pure state, $\rho_{1}=\left|\psi_{1}><\psi_{1}\right|$ then $P\left(\rho_{1}, \rho_{2}\right)=<\psi_{1}\left|\rho_{2}\right| \psi_{1}>$;

4. $P\left(\rho_{1}, \rho_{2}\right)$ is invariant under unitary transformations on the state space;

5. $P\left(\left.\rho_{1}\right|_{\mathcal{A}},\left.\rho_{2}\right|_{\mathcal{A}}\right) \geq P\left(\rho_{1}, \rho_{2}\right)$ for any complete subalgebra of observables $\mathcal{A}$;

6. $P\left(\rho_{1} \otimes \sigma_{1}, \rho_{2} \otimes \sigma_{2}\right)=P\left(\rho_{1}, \rho_{2}\right) P\left(\sigma_{1}, \sigma_{2}\right)$.

Uhlmann's transition probability for mixed states 3

$$
P\left(\rho_{1}, \rho_{2}\right)=\left[\operatorname{Tr}\left(\sqrt{\rho_{1}} \rho_{2} \sqrt{\rho_{1}}\right)^{1 / 2}\right]^{2}
$$

satisfies properties 1-6. The fidelity is defined by $F\left(\rho_{1}, \rho_{2}\right)=P\left(\rho_{1}, \rho_{2}\right)$. A detailed analysis of the structure of the transition probability was hampered by the factors containing square roots in Eq. (位). Due to technical difficulties in the computation of fidelities, few concrete examples of analytic calculations are known. Until recently, all the results were obtained only for finitedimensional Hilbert spaces [12 14]. The first results in an infinite-dimensional Hilbert space were recently obtained by Twamley [9] for the fidelity of two thermal squeezed 
states and by Paraoanu and Scutaru [10] for the case of two displaced thermal states. In [1] Scutaru has developed another calculation method which allowed getting the result for the case of two displaced thermal squeezed states in a coordinate-independent form.

Let $(E, \sigma)$ be a phase space i.e. a vector space with a symplectic structure $\sigma$. Then the commutation relations on $(E, \sigma)$ acting in a Hilbert space $\mathcal{H}$ are defined by a continuous family of unitary operators $\{V(u), u \in E\}$ on $\mathcal{H}$ which satisfy the Weyl relations $[7,8]$ :

$$
V(u) V(v)=\exp \left\{\frac{i}{2} \sigma(u, v)\right\} V(u+v) .
$$

Hence the family $\{V(t u),-\infty<t<\infty\}$ for a fixed $u \in E$ is a group of unitary operators.

Then by the Stone theorem

$$
V(u)=\exp \{i R(u)\}
$$

where $R(u)$ is a selfadjoint operator. From the Weyl relations we have

$$
\begin{aligned}
& \exp \{i t R(u)\} \exp \{i s R(v)\}= \\
& \exp \{i t s \sigma(u, v)\} \exp \{i s R(v)\} \exp \{i t R(u)\}
\end{aligned}
$$

By differentiation and taking $t=s=0$ one obtains

$$
[R(u), R(v)]=-i \sigma(u, v) I .
$$

The operators $\{R(u), u \in E\}$ are called cannonical observables.

The phase space $E$ is of even real dimension $2 n$ and there exist in $E$ symplectic bases of vectors $\left\{e_{j}, f_{j}\right\}_{j=1, \ldots, n}$, i.e. reference systems such that $\sigma\left(e_{j}, e_{k}\right)=\sigma\left(f_{j}, f_{k}\right)=0$ and $\sigma\left(e_{j}, f_{k}\right)=-\sigma\left(f_{k}, e_{j}\right)=$ $\delta_{j k}, j, k=1, \ldots, n$. The coordinates $\left(\xi^{j}, \eta^{j}\right)$ of a vector $u \in E$ in a symplectic basis, $u=\sum_{j=1}^{n}\left(\xi^{j} e_{j}+\eta^{j} f_{j}\right)$, are called symplectic coordinates. The well known coordinate and momentum operators are defined by $Q_{k}=$ $R\left(f_{k}\right)$ and $P_{k}=R\left(e_{k}\right)$ for $k=1,2, \ldots, n$. Then the canonical observables $R(u)$ are linear combinations of the above defined coordinate and momentum operators: $R(u)=\sum_{j=1}^{n}\left(\xi^{j} P_{j}+\eta^{j} Q_{j}\right)$.

There is a one-to-one correspondence between the symplectic bases and the linear operators $J$ on $E$ defined by $J e_{k}=-f_{k}$ and $J f_{k}=e_{k}, k=1, \ldots, n$. The essential properties of these operators are: $\sigma(J u, u) \geq 0$, $\sigma(J u, v)+\sigma(u, J v)=0\left(u, v \in E\right.$ and $J^{2}=-I$, where $I$ denotes the identity operator on $E$ ). Such operators are called complex structures. In the following we shall use the matriceal notations with $u \in E$ as column vectors. Then $\sigma(u, v)=u^{T} J v$ and the scalar product is given by $\sigma(J u, v)=u^{T} v, u, v \in E$. A linear operator $S$ on $E$ is called a symplectic operator if $S^{T} J S=J$. When $S$ is a symplectic operator then $S^{T}$ and $S^{-1}$ are also symplectic operators. The group of all symplectic operators $\operatorname{Sp}(E, \sigma)$ is called the symplectic group of $(E, \sigma)$.
The Lie algebra of $S p(E, \sigma)$ is denoted by $s p(E, \sigma)$ and its elements are operators $R$ on $E$ with the property: $(J R)^{T}=J R$. Hence an operator $R$ on $E$ belongs to $S p(E, \sigma) \cap s p(E, \sigma)$ iff $R^{2}=-I$. If $J$ and $K$ are two complex structures, there exists a symplectic transformation $S$ such that $J=S^{-1} K S$. For any symplectic operator $S$ we can define a new system of Weyl operators $\{V(S u) ; u \in E\}$. Then from a well known result on the unicity of the the systems of Weyl operator up to a unitary equivalence it follows that there exists a unitary operator $U(S)$ on $\mathcal{H}$ such that $V(S u)=U(S)^{\dagger} V(u) U(S)$.

For any nuclear operator $O$ on $\mathcal{H}$ one defines the characteristic function

$$
C F_{u}(O)=\operatorname{TrOV}(u), \quad u \in E
$$

We give the properties of the characteristic function which are important in the following [7]:

$$
\begin{aligned}
& \text { 1. } C F_{0}(O)=\operatorname{Tr} O ; \\
& \text { 2. } C F_{u}\left[V(v)^{*} O V(v)\right]=C F_{u}[O \exp i \sigma(v, u)] \text {; } \\
& \text { 3. } C F_{u}\left(O_{1} O_{2}\right)= \\
& \quad \frac{1}{(2 \pi)^{n}} \int C F_{v}\left(O_{1}\right) C F_{u-v}\left(O_{2}\right) \exp \frac{i}{2} \sigma(v, u) d v ;
\end{aligned}
$$

4. $C F_{S u}(O)=C F_{u}\left(U(S) O U(S)^{\dagger}\right)$.

\section{MULTIMODE THERMAL SQUEEZED STATES}

The multimode thermal squeezed states are defined by the density operators $\rho$ whose characteristic functions are Gaussians 1, 1,8]

$$
C F_{u}(\rho)=\exp \left\{-\frac{1}{4} u^{T} A u\right\} .
$$

where $A$ is a $2 n \times 2 n$ positive definite matrix, called correlation matrix. From the last property of the characterisic function, enumerated above, it follows that:

$$
A_{U(S) \rho U(S)^{\dagger}}=S^{T} A_{\rho} S
$$

Because the correlation matrix A is positive definite it follows 8,15] that there exists $S \in S p(E, \sigma)$ such that

$$
A=S^{T} \mathcal{D} S
$$

where $\mathcal{D}=\left(\begin{array}{cc}D & 0 \\ 0 & D\end{array}\right)$ and $D \geq I$ is a diagonal $n \times n$ matrix. The most general real symplectic transformation $S \in S p(E, \sigma)$ has [8,16] the following structure:

$$
S=O \mathcal{M} O^{\prime}
$$

where

$$
\mathcal{M}=\left(\begin{array}{cc}
M & 0 \\
0 & M^{-1}
\end{array}\right)
$$


and $O, O^{\prime}$ are symplectic and orthogonal $\left(O^{T} O=I\right)$ operators, and where $M$ is a diagonal $n \times n$ matrix. Various particular kinds of such matrices are obtained taking $O, O^{\prime}, \mathcal{D}$ or $\mathcal{M}$ to be equal or proportional to the corresponding identity operator. A pure squeezed state is obtained when $\mathcal{D}=I$. If this condition is not satisfied, the state is a mixed state called thermal squeezed state [17]. When $\mathcal{M}=I$ there is no squeezing and the correspondig states are pure coherent states or thermal coherent states. All these states have correlations between the different modes produced by the orthogonal symplectic operators $O$ and $O^{\prime}$. As a consequence the most general form of a correlation matrix $A$ is given by:

$$
A=O^{\prime T} \mathcal{M} O^{T} \mathcal{D} O \mathcal{M} O^{\prime}
$$

From the property 3 of the characteristic function we have for two density operators $\rho_{1}$ an $\rho_{2}$

$$
\begin{aligned}
& C F_{u}\left(\rho_{1} \rho_{2}\right)=\left[\operatorname{det}\left(\frac{A_{1}+A_{2}}{2}\right)^{-\frac{1}{2}}\right] \\
& \exp \left\{-\frac{1}{4} u^{T}\left[A_{2}-\left(A_{2}-i J\right)\left(A_{1}+A_{2}\right)^{-1}\left(A_{2}+i J\right)\right] u\right\} .
\end{aligned}
$$

When $\rho_{1}=\rho_{2}$ we have

$$
C F_{u}\left(\rho^{2}\right)=(\operatorname{det} A)^{-\frac{1}{2}} \exp \left\{-\frac{1}{4} u^{T}\left(\frac{A-J A^{-1} J}{2}\right) u\right\} .
$$

A state $\rho$ is pure iff $\rho^{2}=\rho$. Then from the equality $C F_{u}\left(\rho^{2}\right)=C F_{u}(\rho)$ it follows that a Gaussian state is pure iff

$$
A=-J A^{-1} J
$$

i.e. a Gaussian state is pure iff $J A \in S p(E, \sigma)$. Analogously, for a mixed state $\rho^{2}<\rho$. Then $C F_{u}\left(\rho^{2}\right)<$ $C F_{u}(\rho)$ and as a consequence $\frac{A-J A^{-1} J}{2}>A$. Hence for any Gaussian state the correlation matrix $A$ must satisfy the folloving restriction

$$
A \leq-J A^{-1} J
$$

\section{THE CHARACTERISTIC FUNCTION OF THE SQUARE ROOT OF A DENSITY MATRIX}

Let us suppose that the characteristic function of the Hilbert-Schmidt operator $\sqrt{\rho}$ of a Gaussian state is, up to a numerical factor, also a Gaussian function with the correlation matrix $\Phi(A)$,

$$
C F_{u}(\sqrt{\rho})=K \exp \left\{-\frac{1}{4} u^{T} \Phi(A) u\right\} .
$$

Then from the equality $\rho=\sqrt{\rho} \sqrt{\rho}$ we obtain

$$
\begin{aligned}
& K^{2}(\operatorname{det}(\Phi(A)))^{-\frac{1}{2}} \\
& \exp \left\{-\frac{1}{4}\left(\frac{\Phi(A)-J \Phi(A)^{-1} J}{2}\right) u\right\}= \\
& \exp \left\{-\frac{1}{4} u^{T} A u\right\} .
\end{aligned}
$$

Hence

$$
K^{2}=\sqrt{\operatorname{det} \Phi(A)}
$$

and

$$
\Phi(A)-J \Phi(A)^{-1} J=2 A .
$$

The last equation has the solution

$$
\Phi(A)=A\left(I+\sqrt{I+(J A)^{-2}}\right) .
$$

This is a new proof of a result obtained in [7]. The advantage of this proof is given by the fact that it does not require the choice of a special basis in $E$. If we take a symplectic basis in E such that $J=\left(\begin{array}{cc}0 & I \\ -I & 0\end{array}\right)$ then $J \mathcal{D}=\mathcal{D} J$ and from this equation and from the equations $A=S^{T} \mathcal{D} S, J S^{T}=S^{-1} J$ it follows that $(J A)^{-2}=$ $S^{-1} \mathcal{D}^{-2} S$. Hence $I+(J A)^{-2}=S^{-1}\left(I-\mathcal{D}^{-2}\right) S$ and

$$
A \sqrt{I+(J A)^{-2}}=S^{T}\left(\sqrt{\mathcal{D}^{2}-I}\right) S
$$

\section{THE GENERAL FORMULA FOR THE FIDELITY OF GAUSSIAN STATES}

The fidelity $F\left(\rho_{1}, \rho_{2}\right)$ for two density operators $\rho_{1}$ and $\rho_{2}$ is defined by

$$
F\left(\rho_{1}, \rho_{2}\right)=\operatorname{Tr}\left(\sqrt{\sqrt{\rho_{1}} \rho_{2} \sqrt{\rho_{1}}}\right) .
$$

As we have seen in section II the characteristic function of a product of operators whose characteristic functions are Gaussians is also a Gaussian. In section III we have obtained a simple formula for the characteristic function of the square root of a density operator whose characteristic function is a Gaussian. Hence we can find a simple formula for the characteristic function of the operator $\sqrt{\rho_{1}} \rho_{2} \sqrt{\rho_{1}}$ :

$$
C F_{z}\left(\sqrt{\rho_{1}} \rho_{2} \sqrt{\rho_{1}}\right)=\sqrt{L} \exp \left\{-\frac{1}{4} z^{T} \mathcal{O} z\right\}
$$

where

$$
\begin{aligned}
& L^{-1}=\operatorname{det} \Phi\left(A_{1}\right)^{-1} \operatorname{det}\left(\frac{\Phi\left(A_{1}\right)+A_{2}}{2}\right) \\
& \operatorname{det}\left(\frac{A_{2}+\Phi\left(A_{1}\right)-\mathcal{U}}{2}\right)
\end{aligned}
$$


where $\mathcal{U}=\left(A_{2}-i J\right)\left(\Phi\left(A_{1}\right)+A_{2}\right)^{-1}\left(A_{2}+i J\right)$, and

$$
\begin{aligned}
& \mathcal{O}=\Phi\left(A_{1}\right)-\left(\Phi\left(A_{1}\right)-i J\right)\left[A_{2}+\Phi\left(A_{1}\right)-\right. \\
& \left.\left(A_{2}-i J\right)\left(\Phi\left(A_{1}\right)+A_{2}\right)^{-1}\left(A_{2}+i J\right)\right]^{-1}\left(\Phi\left(A_{1}\right)+i J\right) .
\end{aligned}
$$

Then applying the result of the preceeding section we can obtain the characteristic function of $\sqrt{\sqrt{\rho_{1}} \rho_{2} \sqrt{\rho_{1}}}$,

$$
\begin{aligned}
& C F_{z}\left(\sqrt{\sqrt{\rho_{1}} \rho_{2} \sqrt{\rho_{1}}}\right)= \\
& {[\operatorname{Ldet} \Phi(\mathcal{O})]^{\frac{1}{4}} \exp \left\{-\frac{1}{4} z^{T} \Phi(\mathcal{O}) z\right\} .}
\end{aligned}
$$

From this formula and the property 1 of the characteristic function we obtain

$$
F\left(\rho_{1}, \rho_{2}\right)=\sqrt{\operatorname{Ldet} \Phi(\mathcal{O})}
$$

We remark that

$$
\operatorname{det} \Phi(\mathcal{O})=\operatorname{det} \mathcal{O} \operatorname{det}\left[I+\sqrt{I+(J \mathcal{O})^{-2}}\right]
$$

In order to simplify the formula for fidelity we observe that

$$
\begin{aligned}
& t_{i j k}=\operatorname{Tr} \rho_{i} \rho_{j} \rho_{k}=\operatorname{det}\left(\frac{A_{i}+A_{j}}{2}\right) \\
& \operatorname{det}\left[\frac{A_{j}+A_{k}-\left(A_{j}-i J\right)\left(A_{i}+A_{j}\right)^{-1}\left(A_{j}+i J\right)}{2}\right],
\end{aligned}
$$

and that $t_{123}=t_{231}=t_{312}$. If we take in this last identity $\Phi\left(A_{1}\right)$ instead of $A_{1}$ then we obtain

$$
\begin{aligned}
& \operatorname{det}\left[\frac{\Phi\left(A_{1}\right)+A_{2}}{2}\right] \\
& \operatorname{det}\left[\frac{A_{2}+\Phi\left(A_{1}\right)-\mathcal{U}}{2}\right] \\
& =\operatorname{det}\left(\frac{A_{1}+A_{2}}{2}\right) \operatorname{det} \Phi\left(A_{1}\right) .
\end{aligned}
$$

Hence we get

$$
L=\left[\operatorname{det}\left(\frac{A_{1}+A_{2}}{2}\right)\right]^{-1} \text {. }
$$

It is not evident from this general formula that the properties 1-6 of the fidelity are valid. Let us consider the most simple one, namely the property $F(\rho, \rho)=1$. In this case it is necessary to prove that $\Phi(\mathcal{O})=A$. We can choose the complex structure $J$ to commute with the correlation matrix $A: J A=A J$. Then all operations in the formula which gives $\mathcal{O}$ as a function of $A$ and $J$ can be performed and the result is: $\mathcal{O}=\frac{A+A^{-1}}{2}$ and $\Phi(\mathcal{O})=A$. The next property which we shall discuss is the property 3 which in the case of Gaussian states becomes [1]: $F\left(\rho_{1}, \rho_{2}\right)=\left(\frac{A_{1}+A_{2}}{2}\right)^{-\frac{1}{2}}$. We shall prove that $\mathcal{O}=A_{1}$ when $\rho_{1}$ is a pure state. First we remark that $\Phi\left(A_{1}\right)=A_{1}$ and that there is a symplectic transformation such that $A_{1}=S^{T} S$. Then $\mathcal{O}=S^{T}\left\{I-2 P_{-}\left[2 I-4 P_{+} \mathcal{X}^{-1} P_{-}\right] 2 P_{+}\right\} S$ where $P_{+}=\frac{I+i J}{2}, P_{-}=\frac{I-i J}{2}$ and $\mathcal{X}=\left(S^{T}\right)^{-1} A_{2} S^{-1}+I$. Evidently $P_{+}$and $P_{-}$are an orthogonal decomposition of the unit operator: $P_{+}^{2}=P_{+}, P_{-}^{2}=P_{-}, P_{+} P_{-}=P_{-} P_{+}=0$ and $P_{+}+P_{-}=I$. As a consequence of the orthogonality we obtain $\mathcal{O}=S^{T} S=A_{1}$. Then $\Phi(\mathcal{O})=\Phi\left(A_{1}\right)=A_{1}=$ $S^{T} S$ and $\operatorname{det}(\Phi(\mathcal{O}))=\operatorname{det}\left(S^{T}\right) \operatorname{det}(S)=1$.

\section{THE ONE MODE CASE}

In [1] we have obtained an expression for the fidelity in the one mode case. This formula can be reobtained as a consequence of the above general formula. In the one mode case all matrices are $2 \times 2$ matrices. For a $2 \times 2$ matrix $\mathcal{O}$ we have

$$
\Phi(\mathcal{O})=\epsilon \mathcal{O},
$$

where $\epsilon=1+\sqrt{1-\frac{1}{\operatorname{det} \mathcal{O}}}$ and $\operatorname{det} \Phi(\mathcal{O})=(\sqrt{\operatorname{det} \mathcal{O}}+$ $\sqrt{\operatorname{det} \mathcal{O}-1})^{2}$. From these considerations it follows that

$$
F\left(\rho_{1}, \rho_{2}\right)=\frac{2}{\sqrt{\operatorname{det}\left(A_{1}+A_{2}\right)}(\sqrt{\operatorname{det} \mathcal{O}}-\sqrt{\operatorname{det} \mathcal{O}-1})} .
$$

Thus it is sufficient to compute $\operatorname{det} \mathcal{O}$. We shall denote by $\mathcal{P}$ the product $\left(\operatorname{det} A_{1}-1\right)\left(\operatorname{det} A_{2}-1\right)$. After simple but long computations we obtain

$$
\operatorname{det} \mathcal{O}=1+\frac{\mathcal{P}}{\operatorname{det}\left(A_{1}+A_{2}\right)},
$$

which gives the result of [1]

$$
F\left(\rho_{1}, \rho_{2}\right)=\frac{2}{\sqrt{\operatorname{det}\left(A_{1}+A_{2}\right)+\mathcal{P}}-\sqrt{\mathcal{P}}} .
$$

\section{MULTIMODE THERMAL STATES CASE}

In the case of two thermal states with correlation matrices $A_{i}=\mathcal{D}_{i}$ with $i=1,2$ we have $A_{i} J=J A_{i},(i=1,2)$ and $A_{1} A_{2}=A_{2} A_{1}$. Then $\Phi\left(A_{i}\right)=A_{i}+\sqrt{A_{i}^{2}-I}$, $(i=1,2)$. Hence

$$
\mathcal{O}=\left(A_{1}+A_{2}\right)^{-1}\left(A_{1} A_{2}+I\right)
$$

and

$$
\Phi(\mathcal{O})=\frac{\left(A_{1}+A_{2}\right)}{\left(A_{1} A_{2}+I\right)-\sqrt{\left(A_{1}^{2}-I\right)\left(A_{2}^{2}-I\right)}}
$$

Finally 
$F\left(\rho_{1}, \rho_{2}\right)=\sqrt{\operatorname{det}\left(\frac{2}{\left(A_{1} A_{2}+I\right)-\sqrt{\left(A_{1}^{2}-I\right)\left(A_{2}^{2}-I\right)}}\right)}$

which is the product of the fidelities of the corresponding one-mode thermal states [1, [7, 8 ]

\section{CONCLUSIONS}

In this paper we have provided a general formula for the calculation of the fidelity of two Gaussian states. It is shown that, in the particular case of a single mode, this formula reproduces the results already known in the literature, and in the case of multimode thermal states it yields a compact expression with a direct interpretation.

\section{ACKNOWLEDGMENTS}

The second author acknowledges a partial financial support from the Grant Agency of the Romanian Academy.

[1] H. Scutaru, J. Phys. A: Mat. Gen. 31, 3659 (1998).

[2] D. Bures, Trans. Am. Math. Soc. 135, 199 (1969).

[3] A. Uhlmann, Rep. Math. Phys. 9, 273 (1976).

[4] R. Josza, J. Mod. Opt. 41, 2314 (1994).

[5] W. K. Wooters and W. H. Zurek, Nature 299, 802 (1982).

[6] A. S. Holevo, M Sohma, and O. Hirota, Phys. Rev. A 59, 1820 (1999); A. S. Holevo, quant-ph/9809022; Gh.-S. Paraoanu and H. Scutaru, in preparation.

[7] A. S. Holevo, Problemy Peredachi Informacii 6 , 44 (1973); A. S. Holevo, IEEE Trans. Inf. Theory IT21, 533 (1975); A. S. Holevo, Theor. Math. Fiz (Russian) 13, 184 (1979); A. S. Holevo, Probabilistic and Statistical Aspects of Quantum Theory, (North Holland, Amsterdam, 1982), Chap 5.

[8] H. Scutaru, Phys. Lett. A 141, 223 (1989); H. Scutaru, Phys. Lett. A 167, 326 (1992); H. Scutaru, Phys. Lett. A 200, 91 (1995); H. Scutaru, J. Math. Phys 39, 6403 (1999).

[9] J. Twamley, J. Phys. A 29, 3723 (1996).

[10] Gh.-S. Paraoanu and H. Scutaru, Phys. Rev. A 58, 869 (1998).

[11] Gh.-S. Paraoanu and H. Scutaru, in preparation.

[12] M. Hübner, Phys. Lett. A 163, 239 (1992).

[13] M. Hübner, Phys. Lett. A bf 179, 226 (1993).

[14] J. Dittman, J. Geom. Phys. 13, 203 (1994).

[15] G. B. Folland, Harmonic analysis in phase space, (Princeton University Press, Princeton, 1989), page 177, Proposition 4.22 .
[16] R.. Balian, C. De Dominicis and C. Itzykson, Nuclear Physics 67, 609 (1965).

[17] H. Ezawa, A. Mann, K. Nakamura and M. Revzen, Ann. Phys. (N.Y.) 209, 216 (1991). 